\title{
Age and metallicity of the bulges in lenticular galaxies
}

\author{
Olga K. Sil'chenko ${ }^{1}$ \\ ${ }^{1}$ Sternberg Astronomical Institute, Moscow 119991, Russia \\ email: olga@sai.msu.su
}

\begin{abstract}
Panoramic spectroscopy of the sample of 80 nearby lenticular galaxies is presented. The SSP-equivalent ages, $[\mathrm{Z} / \mathrm{H}]$, and $[\mathrm{Mg} / \mathrm{Fe}]$ are determined through the Lick indices $\mathrm{H} \beta$, $\mathrm{Mgb}$, and $\langle\mathrm{Fe}\rangle$ separately for the nuclei and for the bulges. About a half of the sample contain chemically distinct nuclei, more metal-rich and younger than the bulges. The statistics of stellar population properties for the nearby S0s is discussed.

Keywords: galaxies: elliptical and lenticular, cD; galaxies: bulges; galaxies: evolution
\end{abstract}

\section{Introduction}

S0 galaxies are perhaps the only class of nearby galaxies which is formed rather recently. Observations of clusters at various redshifts have shown that the fraction of S0s there rose from $0-10 \%$ to $50 \%-60 \%$ between the lookback times of 6 and 2 Gyr (Fasano et al. 2000, Desai et al. 2007). The common point of view is that S0s are transformed of spirals due to some external action related to dense environment and/or hot intergalactic medium. Theoreticians propose a lot of mechanisms for such transformation: ram pressure by intracluster medium, tidal stripping and heating, harassment, minor merger... Probably, different mechanisms may play role in different environments, because in fact you can meet lenticular galaxies from field to clusters. Most mechanisms lead to gas concentration in the very centers of the galaxies at the moment of their transformation. It means that we can expect secondary star formation bursts and intermediate-age stellar populations in the nuclei of nearby S0s. Also, the statistics on bulge-disk luminosity ratios show that nearby S0s have on average more massive bulges than nearby spirals (e.g. Trujillo et al. 2002), so their transformation must include some process of bulge growth. It means that the bulges must have suffered some rejuvenation during the last $5 \mathrm{Gyr}$, and their stellar populations may be on average younger than those in nearby ellipticals. So there are good reasons to extract S0 galaxies from the wider samples of 'early-type' and 'red-sequence' galaxies and to search for particular properties of their stellar populations.

\section{Sample}

We consider a sample of 80 nearby S0s over the wide range of luminosity. Our sample is not complete but rather representative. We have undertaken a retrieval over the HYPERLEDA with the following restrictions:

- $v_{r}<3000 \mathrm{~km} / \mathrm{s}$;

- $-3 \leqslant t \leqslant 0$, where $t$ is the numerical morphological-type indicator;

- $\delta(2000.0)>0$;

- $B_{T}^{0} \leqslant 13.0$.

Strong Seyfert nuclei or nuclear star bursts were excluded. The search gave us a list of 148 S0s, with 50 Virgo members among them. We have observed 66 targets from 
this list, including 10 Virgo members. Also a few more distant luminous galaxies as well as nearby fainter ones were added to the sample to expand the range of luminosities. By following NOG survey of groups (Giuricin et al. 2000), we put our galaxies into four types of environments: those belonging to clusters (Virgo and Ursa Major), group central galaxies (the brightest galaxies in groups), group second-rank members, and field galaxies. Finally, we have 13, 22, 33, and 12 galaxies in every class.

The novelty of our approach to bulges is that we do not use aperture spectral data focused onto the centers of galaxies. Inspired by the panoramic spectroscopy benefits, we consider separately unresolved stellar nuclei and bulges taken as rings between $R=4^{\prime \prime}$ and $R=7^{\prime \prime}$; the latter $R$ corresponds to $1.3 \mathrm{kpc}$ for the most distant galaxies.

\section{Observations and data reduction}

All the observations have been made with the integral-field spectrograph of the Russian 6-m telescope, Multi-Pupil Field/Fiber Spectrograph (MPFS)(Afanasiev et al. 2001). Details of the observations and data reductions can be found in (Sil'chenko 2006).

\section{Results}

Age $T$, total metallicity $[\mathrm{Z} / \mathrm{H}]$, and magnesium-to-iron ratio $[\mathrm{Mg} / \mathrm{Fe}]$ are determined for the nuclei and for the bulges by using models of stellar populations by Thomas et al. (2003). SSP approach (single-burst one-metallicity population) is applied. The $T$ and $[\mathrm{Z} / \mathrm{H}]$ are obtained first, by confronting $\mathrm{H} \beta$ to $[\mathrm{MgFe}]=(\mathrm{Mgb}\langle\mathrm{Fe}\rangle)^{1 / 2}$ (insensitive to $\mathrm{Mg} / \mathrm{Fe}$ ratio), and the abundance ratio $[\mathrm{Mg} / \mathrm{Fe}]$ is determined by confronting $\langle\mathrm{Fe}\rangle$ to Mgb for a given $T$.

By starting our study of nuclei and bulges of early-type galaxies with the MPFS in 1989, we discovered immediately that the (unresolved) nuclei differ strongly from the surrounding bulges, namely, the nuclei demonstrated stronger metal absorption lines (Sil'chenko et al. 1992). Now, with our sample of 80 lenticular galaxies observed with the MPFS, we can state that about half of them have chemically distinct nuclei. If we select the galaxies where the nuclei are more metal-rich than the bulges by +0.3 dex and more, we come to the estimate of $42 \%$. The low limit put by us onto the $[\mathrm{Z} / \mathrm{H}]$ difference to select chemically distinct nuclei is rather conservative and is defined by the current estimates of the metallicity gradients in spheroidal galaxies which are about $0.2-0.3$ dex per radius dex. The chemically distinct nuclei have the same metallicity drop inside the radius equal to one or two resolved spatial elements. By comparing the subsample of the S0s with the chemically distinct nuclei to the total sample of nearby S0s (Fig. 1), we see that the chemically distinct nuclei are always younger than the surrounding bulges. The mean age difference between the chemically distinct nuclei and their surrounding bulges is $2.8 \mathrm{Gyr}$ while that between the nuclei and the bulges for the whole sample is only 1 Gyr. The age distribution of the chemically distinct nuclei peaks strongly at $T=2$ Gyr whereas the total nuclear age distribution is rather flat between $T=1$ and $T=12$ Gyr.

So it is necessary to separate the nuclei and the bulges before making any conclusions about the properties of any of them. Nuclei and bulges have quite different evolution and differ as concerning their present stellar populations. Further we consider the bulges as taken in the rings between the radii of $4^{\prime \prime}$ and $7^{\prime \prime}$ - within the areas of bulge domination but beyond those affecting by the nuclei influence due to seeing effects.

Initially, we distinguished 4 types of environments. However, at all diagnostic diagrams 'index vs index' the cluster S0s occupy just the same area as the group center galaxies, and group second-rank members are indistinguishable from the field galaxies. So we unite 

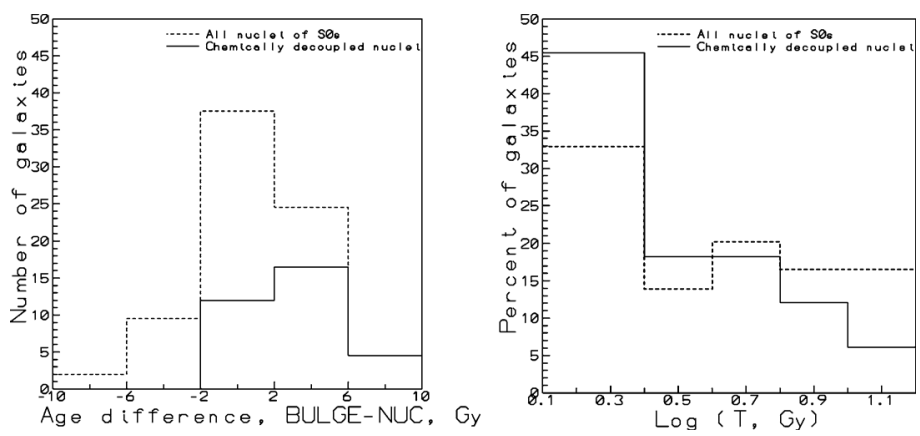

Figure 1. The results on mean stellar ages for the chemically distinct nuclei in comparison to the total sample of the nuclei in lenticular galaxies. $(a)$ the histogram of the stellar age difference between the nuclei and the bulges; the chemically distinct nuclei are always younger than the surrounding bulges; $(b)$ the distributions of the absolute values of the SSP-equivalent ages for the nuclei; the chemically distinct nuclei are separately shown by a solid line.

all the galaxies in two big groups: S0s in dense environments, namely, in clusters and group centers, and S0s in sparse environments, namely, in field and off-centered in groups. The median stellar ages of the bulges are 4.0 Gyr in sparse environments and 6.7 Gyr in dense environments; the corresponding estimates for the nuclei are 2.4 and $4.1 \mathrm{Gyr}$, so the nuclei are on average younger than the bulges in any types of environments. To test if the age difference is indeed due to environment density, we must analyze age correlation with the stellar velocity dispersion. Such relations are well-studied for elliptical galaxies. We can compare our results obtained for the bulges of S0s, first of all, with these data.

But as we separate the nuclei and the bulges when studying the properties of stellar populations, similarly, we measure stellar velocity dispersion separately in the very centers and in the bulges, at $R \sim 5^{\prime \prime}$. Indeed, the results reveal again distinction between the nuclei and the bulges: the stellar velocity dispersion in the nuclei is typically larger than that in the bulges, with the difference up to $70-80 \mathrm{~km} / \mathrm{s}$, the mean being 27 $\mathrm{km} / \mathrm{s}$. To make a fair comparison, we confront the stellar population parameters to the bulge stellar velocity dispersion. This reduces our sample to 52 galaxies, because accurate stellar velocity dispersion mapping in the full mass range over the full field of view becomes possible only with the spectral resolution of $3 \AA$, after 2002. Below we analyze the correlations over the range of $\sigma_{*}=50-200 \mathrm{~km} / \mathrm{s}$.

In Fig. 2 we show dependencies of $T,[\mathrm{Z} / \mathrm{H}]$, and $[\mathrm{Mg} / \mathrm{Fe}]$ on $\log \sigma_{*}$, in comparison with the recent results for early-type galaxies in various types of environment from Thomas et al. (2005), Nelan et al. (2005), and Howell (2005) which have been obtained for $\sigma_{*}>$ $100 \mathrm{~km} / \mathrm{s}$ typically. One can see that the age and metallicity dependencies on $\log \sigma_{*}$ are consistent with those for elliptical galaxies. The age correlates with $\log \sigma_{*}$, and the metallicity does not (Howell 2005). However, the correlation of the abundance ratio $[\mathrm{Mg} / \mathrm{Fe}]$ with $\log \sigma_{*}$ is the strongest, and the slope of the regression is much steeper than the slope which is consistently found by several groups of investigators for elliptical galaxies. What does it mean? If we treat the correlation of $[\mathrm{Mg} / \mathrm{Fe}]$ with the mass of spheroid as an evidence for more effective (and brief) star formation in deeper potential well, we may suggest that the relation found for the bulges of S0s is fundamental for some early formation process. Consequently, the flatter relation for ellipticals may be a result of later 'dry mergers' which increase the mass of spheroids leaving the properties of stellar population corresponding to the masses of smaller progenitors. 



Figure 2. Correlations between the stellar population parameters and the logarithm of the local stellar velocity dispersion for the bulges of nearby lenticular galaxies. Local relations for various samples of early-type galaxies from the literature are also shown. (a) the SSP-equivalent age vs $\log \sigma_{*} ;(b)$ the total metallicity vs $\log \sigma_{*} ;(c)$ the magnesium-to-iron ratio vs $\log \sigma_{*}$.

\section{Conclusions}

Unresolved stellar nuclei in early-type disk galaxies have their own evolution: $42 \%$ of nearby S0s have $\Delta[\mathrm{Z} / \mathrm{H}]($ nuc-bul $)=+0.3$ dex and more and the mean $\Delta T($ nuc-bul $)=-2.8$ Gyr. The difference of the stellar velocity dispersion $\Delta \sigma_{*}$ (nuc-bul) may reach $70 \mathrm{~km} / \mathrm{s}$, with the mean of $27 \mathrm{~km} / \mathrm{s}$. So to study bulges, one needs to measure off-nuclear zones.

In sparse environments there is a much larger spread of ages than in clusters and group centers. However when correlating the ages, metallicities, and $\mathrm{Mg} / \mathrm{Fe}$ ratio with the stellar velocity dispersion, there is no separation due to the environment type. The SSP-equivalent ages correlate with the local stellar velocity dispersions, while the SSPequivalent metallicities do not. But the age spread rises to larger $\sigma_{*}$ - unlike ellipticals (Caldwell et al. 2003). The abundance ratio $[\mathrm{Mg} / \mathrm{Fe}]$ correlates strongly with the stellar velocity dispersion, and the relation slope is much steeper for $\mathrm{S} 0$ bulges than for ellipticals.

\section{Acknowledgements}

This study has been supported by the grants of the Russian Foundation for Basic Researches (RFBR), nos. 01-02-16767, 04-02-16987, and 05-02-19805-MFa. My attendance at the Symposium is due to the IAU grant and to the RFBR travel grant no. 07-02-08314.

\section{References}

Afanasiev, V. L., Dodonov, S. N., \& Moiseev, A. V. 2001, in: L. P. Osipkov \& I. I. Nikiforov (eds.), Stellar dynamics: from classic to modern, (Saint Petersburg: SPb Univ. press), p. 103

Caldwell, N., Rose, J. A. \& Concannon, K. D. 2003, AJ 125, 2891

Desai, V., Dalcanton, J. J., Aragón-Salamanca, A., et al. 2007, ApJ 660, 1151

Fasano, G., Poggianti, B. M., Couch, W. J., Bettoni, D., Kjaergaard, P., \& Moles, M. 2000, ApJ 542,673

Giuricin, G., Marinoni, C., Ceriani, L., \& Pisani, A. 2000, ApJ 543, 178

Howell, J. H. 2005, AJ 130, 2065

Nelan, J. E., Smith, R. J., Hudson, M. J., et al. 2005, ApJ 632, 137

Sil'chenko, O. K. 2006, ApJ 641, 229

Sil'chenko, O. K., Afanasiev V. L., \& Vlasyuk V. V. 1992, AZh 69, 1121

Thomas, D., Maraston, C., \& Bender, R. 2003, MNRAS 339, 897

Thomas, D., Maraston, C., Bender, R., Mendes de Oliveira, C. 2005, ApJ 621, 673

Trujillo, I., Asension Ramos, A., Rubino-Martín, J. A., et al. 2002, MNRAS 333, 510 